\title{
Redução de inóculo de Aphelenchoides besseyi em sementes de Brachiaria brizantha tratadas com óleos essenciais
}

\author{
Inoculum reduction of Aphelenchoides besseyi in Brachiaria brizantha seeds \\ treated with essential oils
}

\begin{abstract}
Thalita Suelen Avelar Monteiro ${ }^{I^{*}}$ Érica das Graças Carvalho Nasu ${ }^{\mathrm{I}}$ Cynthia Pires Guimarães $^{\mathrm{II}}$

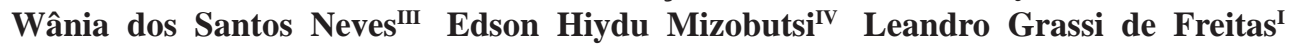

RESUMO

Óleos essenciais foram testados com o objetivo de controlar Aphelenchoides besseyi em sementes de Brachiaria brizantha $c v$. 'Marandu'. Sementes naturalmente infestadas por A. besseyi foram tratadas com óleos essenciais de mostarda (Brassica nigra), canela (Cinnamomum zeylanicum), verbena (Lippia citriodora) e Bioprotector ${ }^{\circledR}$ (produto comercial composto por extratos e óleos essenciais derivados de plantas), nas concentrações de 0,5\% e 1,0\%. Avaliou-se também o efeito dos tratamentos após aplicação direta nos juvenis (ensaio in vitro). Todos os óleos tiveram sua efetividade reduzida no ensaio em semente (in vivo) em relação ao ensaio in vitro. Os tratamentos não erradicaram o nematoide das sementes, sendo que os óleos essenciais de canela e verbena, nas concentrações $0,5 \%$ e 1,0\%, Bioprotector $^{\circledR}$ a 1,0\% e óleo de mostarda a 1,0\% reduziram o nível populacional do nematoide em sementes contaminadas. O óleo de mostarda a 1,0\% destacou-se dos demais por ter apresentado ação nematicida sem alterar a germinação das sementes.

Palavras-chave: controle alternativo, nematoides, sementes de forrageira, tratamento de semente.

\section{ABSTRACT}

Essencial oils were tested to control Aphelenchoides besseyi in Brachiaria brizantha cv. 'Marandu' seeds. Seeds naturally infested with $A$. besseyi were treated with essential oils of mustard (Brassica nigra), cinnamon (Cinnamomum zeylanicum), verbena (Lippia citriodora) and Bioprotector ${ }^{\circledR}$ (a commercial product based on formulation of plant extracts and essential oils), in concentrations of $0.5 \%$ and $1.0 \%$. The effects of the same treatments after direct application on juveniles nematodes were evaluated in vitro. All treatments were less effective to control the nematode in the seeds (in vivo) comparing to the in vitro test. The treatments did not eradicate the nematode from the seeds. However, the essential oils of cinnamon and verbena (in both concentrations), mustard (1.0\%), as well as Bioprotector ${ }^{\circledR}(1.0 \%)$, reduced the seed population of A. besseyi. Mustard oil (1.0\%) stood out for its nematicide action with no effect on seed germination.

Key words: alternative control, forage seeds, nematodes, seed treatment.

\section{INTRODUÇÃO}

O Brasil é o maior produtor, exportador e possui o maior mercado consumidor de sementes forrageiras tropicais (SOUZA, 2001), exportando-as para mais de 20 países, o que movimenta anualmente aproximadamente 250 milhões de dólares e gera cerca de 50 mil empregos no país (VECHIATO \& APARECIDO, 2008). Apesar de economicamente expressivo, esse mercado encontra-se ameaçado pela presença de patógenos (MARCHI et al., 2007).

A despeito da inexistência de pesquisas que quantifiquem os danos causados às pastagens por fitonematoides, há relatos de que estes podem apresentar efeito negativo sobre a produção de matéria seca, qualidade das sementes, persistência e regeneração natural das forrageiras em campo (BERNARD et al., 1998; PEDERSON \& QUESSENBERRY, 1998).

Além disso, nematoides dos gêneros Aphelenchoides FISCHER (1894) e Ditylenchus FILIPJEV (1936) têm implicações de natureza

\footnotetext{
'Universidade Federal de Viçosa (UFV), 36570-900, Viçosa, MG, Brasil. E-mail: thalita.monteiro@ufv.br. *Autor para correspondência.

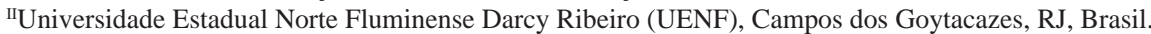

III Empresa de Pesquisa Agropecuária de Minas Gerais (Epamig), Prudente de Morais, MG, Brasil.

${ }^{\text {IV } U n i v e r s i d a d e ~ E s t a d u a l ~ d e ~ M o n t e s ~ C l a r o s ~(U n i m o n t e s), ~ J a n a u ́ b a, ~ M G, ~ B r a s i l . ~}$
} 
quarentenária, dificultando o comércio internacional. De acordo com MARCHI et al. (2007), a presença desses patógenos é limitante para o comércio de sementes de Brachiaria spp. e Panicum spp. , visto que a ocorrência desses nematoides, independentemente da população, muitas vezes, é suficiente para restringir a venda para alguns países importadores. Dessa forma, é necessário a sua erradicação ou controle, para evitar que proporcionem prejuízos à agricultura, bem como sua dispersão para regiões onde ainda não ocorrem.

A utilização de extratos ou óleos essenciais tem sido relatada como uma alternativa promissora no controle de nematoides (KONG et al., 2007; MONFORT et al., 2007; ANDRÉS et al., 2012; BALDIN et al., 2012). Óleos essenciais são tidos como substâncias voláteis naturais, encontrados em uma ampla variedade de plantas, sendo misturas complexas resultantes da interação entre diferentes classes de compostos, como terpenos, monoterpenos, sesquiterpenos, compostos aromáticos, fenóis, aldeídos, cetonas, alcoois e ésteres (BAKKALI et al., 2008; ANDRÉS et al., 2012)

Alguns metabólitos secundários de plantas, presentes nos óleos essenciais e extratos, não exercem função direta no crescimento e desenvolvimento vegetal. Esses compostos são sintetizados pelas plantas para exercerem atividade de adaptação ambiental, atração de polinizadores e fitoproteção (TAIZ \& ZEIGHER, 2004), inclusive contra nematoides (FERRAZ et al., 2010).

No Brasil, estudos que tratam do controle de nematoides em sementes com óleos essenciais são escassos. Sendo assim, o presente trabalho buscou avaliar a eficácia de óleos essenciais e de um produto a base de extratos e óleos vegetais no controle do nematoide Aphelenchoides besseyi CHRISTIE (1942) e na qualidade fisiológica de sementes de Brachiaria brizantha Hochst Stapf cv. 'Marandu'.

\section{MATERIAL E MÉTODOS}

Sementes de Brachiaria brizantha cv. 'Marandu', oriundas de campo de produção localizado no município de Chapada Gaúcha, MG, naturalmente infestadas por Aphelenchoides besseyi, foram submetidas a nove tratamentos, num esquema fatorial de $(4 \times 2)+1$, em delineamento inteiramente casualizado, com cinco repetições, contendo $10 \mathrm{~g}$ de sementes cada. Os tratamentos consistiram de T1: produto comercial Bioprotector (a base de extratos e óleos vegetais), T2: óleo essencial de canela (Cinnamomum zeylanicum Blume.), T3: óleo essencial de verbena (Verbena officinalis L.), T4: óleo essencial de mostarda (Brassica nigra (L.) Koch.), todos diluídos em água, nas concentrações de 0,5\% (v/v) e 1\% (v/v), e T5: tratamento testemunha (água).

Avaliação do controle de Aphelenchoides besseyi nas sementes

As sementes foram imersas nas soluções, referentes a cada tratamento, por 30 minutos e em seguida trituradas em liquidificador em baixa potência por 20 segundos (BRASIL, 2009). A suspensão resultante foi colocada em Funil de Baermann modificado (CHRISTIE \& PERRY, 1951). Posteriormente, foram recolhidas as suspensões dos funis e vertidas em peneira de 125 mesh $(0,150 \mathrm{~mm}$ de abertura), sobre outra de 500 mesh $(0,025 \mathrm{~mm}$ de abertura) e realizada a contagem dos nematoides em Câmara de Peters, sob microscópio ótico.

Avaliação da qualidade fisiológica das sementes

As sementes, submetidas aos tratamentos, foram lavadas em água corrente para retirada do excesso da solução. A seguir, foram dispostas em placas de Petri, sobre duas folhas mata-borrão (BRASIL, 2009), em número de 25 para cada repetição, totalizando cinco placas por tratamento. Posteriormente, foram armazenadas em câmara tipo BOD a $25^{\circ} \mathrm{C}$ durante 21 dias, sob regime alternado de luz (12h de luz e $12 \mathrm{~h}$ de escuro).

$\mathrm{O}$ vigor (V) foi avaliado pela contagem das sementes germinadas ao 7o dia e a germinação (PG) ao 21ํ dia, conforme recomendado por BRASIL (2009).

\section{Avaliação do controle de Aphelenchoides besseyi in} vitro

Os nematoides foram extraídos de sementes por meio de trituração em liquidificador, em seguida suspensões calibradas de 100 juvenis foram colocadas em placas de petri e receberam os tratamentos por 30min. Os nematoides foram recolhidos em peneira de 500 mesh $(0,025 \mathrm{~mm}$ de abertura) e a suspensão foi colocada em Funil de Baermann modificado (CHRISTIE \& PERRY, 1951). Em seguida, foi realizada a contagem dos nematoides em Câmara de Peters, sob microscópio ótico.

Análise de dados

As variáveis número de nematoides, percentagens de germinação e vigor das sementes foram classificadas como variáveis discretas. Assim, testou-se, através do procedimento GLM (General Linear Models), a aditividade, por meio da análise de covariância dos valores preditos ao quadrado. Testou-se também a normalidade, pelo procedimento 
univariate, com a estatística W (Shapiro-Wilk) e a homogeneidade de variância pelo teste de Bartlett. Uma vez que o teste de normalidade não foi atendido, para a variável número de nematoides nas sementes, aplicou-se o teste de Kruskal-Wallis e, quando significativo, as médias foram comparadas pelo teste de $\mathrm{P}$ contraste. Os dados de germinação, vigor e controle de nematoides (teste in vitro) foram submetidos à análise de variância e, quando o teste $\mathrm{F}$ foi significativo, as médias foram comparadas pelos testes Scott Knott $(\mathrm{P}<0,05)$ e Dunnet $(\mathrm{P}<0,05)$.

\section{RESULTADOS E DISCUSSÃO}

Avaliação do controle de Aphelenchoides besseyi nas sementes

Todos os tratamentos, na concentração de $1,0 \%$ e os óleos de canela e de verbena, na concentração de $0,5 \%$, proporcionaram redução do número de juvenis de Aphelenchoides besseyi nas sementes (Tabela 1). O óleo de mostarda $(1,0 \%)$ controlou $44 \%$ dos nematoides (Tabela1). NEVES et al. (2008) também relataram a atividade nematicida do óleo de mostarda (Brassica campestris L.). Esses autores obtiveram 100\% de mortalidade de Meloidogyne javanica (Treub) Chitwood em tratamentos que receberam esse óleo, bem como naqueles que continham alil isotiocianato, composto presente na mostarda.

O tratamento com Bioprotector ${ }^{\circledR}$ a $1,0 \%$ promoveu redução de 52\% no número de juvenis de A. besseyi quando comparado à testemunha (Tabela 1). Esse produto, de composição não revelada, já foi

Tabela 1 - Número de juvenis de Aphelenchoides besseyi presentes em $10 \mathrm{~g}$ de sementes de Brachiaria brizantha cv. Marandu submetidas tratamentos com óleos essenciais e o produto Bioprotector ${ }^{\circledR}$.

\begin{tabular}{lcc}
\hline \multirow{2}{*}{ Tratamentos } & \multicolumn{3}{c}{$0,5 \%$} & $1,0 \%$ \\
\hline Bioprotector $^{\circledR}$ & $492,9 \mathrm{aC}^{\mathrm{NS}}$ & $342,0 \mathrm{aB}$ \\
Canela & $222,9 \mathrm{bA}^{*}$ & $123,2 \mathrm{aA}^{*}$ \\
Verbena & $255,7 \mathrm{aA}^{*}$ & $419,8 \mathrm{bC}^{*}$ \\
Mostarda & $484,9 \mathrm{aB}^{\mathrm{NS}}$ & $401,6 \mathrm{aAB}^{*}$ \\
Controle & \multicolumn{3}{c}{712,0} \\
\hline
\end{tabular}

Médias seguidas de mesma letra, maiúscula na coluna e minúscula na linha, não diferem entre si pelos testes de Kruskall Wallis e Wilcoxon, respectivamente, em nível de $5 \%$ de probabilidade.

Médias seguidas de * diferem do tratamento controle em nível de $5 \%$ de probabilidade e médias seguidas de ${ }^{\mathrm{NS}}$ não diferem do tratamento controle, ambos pelo teste de Dunnet. testado mostrando-se eficiente no controle de oídio (Sphaerotheca fuliginea (Schltdl.) POLLACCI, 1913) em pepino (SILVA JUNIOR et al., 2007), porém relatos de sua ação nematicida são escassos.

O óleo essencial de verbena proporcionou redução no número de nematoides na ordem de $64 \%(0,5 \%)$ e de $41 \%(1,0 \%)$ (Tabela 1). Esse efeito provavelmente se deve à ação de metabólitos secundários do grupo dos flavonoides, do citral, do geraniol e do citronelol (SANGWAN et al., 1985). Flavonoides, presentes também em Artemisia dracunculus L., apresentam comprovada atividade antihelmíntica. TIMCHENKO \& MAIKO (1989), ao aplicarem material vegetal de A. dracunculus no solo, obtiveram a redução da população de Ditylenchus dipsaci (Kühn) Filipjev em 90-96\%. SANGWAN et al. (1985) constataram a atividade nematicida dos óleos essenciais de três gramíneas e de seus constituintes (citral, geraniol e citronelol), sobre juvenis de segundo estádio de quatro espécies de nematoides (Anguina tritici (STEINBUCH, 1799) FILIPJEV, 1936, Tylenchulus sp., M. javanica e Heterodera avenae (WOLLENWEBER,1924)).

O tratamento com óleo de canela reduziu em 83\% o número de juvenis de $\boldsymbol{A}$. besseyi na concentração de $1,0 \%$ e em $69 \%$ na de $0,5 \%$ (Tabela 1 ). O efeito nematicida desse óleo pode ser atribuído à ação química de alguns compostos, como o eugenol e ácido cinâmico, presentes nessa planta (KONG et al., 2007). Tal proposição corrobora os estudos de BALA \& SUKUL (1987), que constataram redução do número de galhas e juvenis de $\boldsymbol{M}$. incognita, além de promoção do crescimento vegetal, após a pulverização de plantas de quiabo com emulsão de eugenol.

A não erradicação dos nematoides deve-se à sua localização no interior das sementes, entre a casca e o endosperma, dificultando a ação dos produtos, o que foi confirmado pelo teste in vitro, em que todos os tratamentos, com exceção do óleo de mostarda, controlaram 100\% dos juvenis de $\boldsymbol{A}$. besseyi (Tabela 2). Apesar de não ter promovido a erradicação dos nematoides in vitro, o óleo de mostarda controlou 68,3 e 78,5\% dos juvenis, e também teve sua efetividade reduzida quando aplicado nas sementes, especialmente na menor concentração que diferiu da testemunha no teste in vitro (Tabela 2), mas não quando aplicado nas sementes (Tabela 1). Isso está de acordo com os trabalhos de GARCIA \& TENENTE (2001) que, ao tratarem sementes de Panicum maximum Jacq. com produtos químicos, conseguiram apenas o controle de $\boldsymbol{A}$. besseyi, e de TENENTE \& MARQUES (1983), que conseguiram erradicar $\boldsymbol{A}$. besseyi quando foram removidas as partes externas das sementes de $\boldsymbol{P}$. maximum. 
Tabela 2 - Número de juvenis de Aphelenchoides besseyi ativos após a aplicação de diferentes tratamentos com óleos essenciais e o produto Bioprotector ${ }^{\circledR}$, em 100 juvenis por amostra.

\begin{tabular}{|c|c|c|}
\hline \multirow{2}{*}{ Tratamentos } & \multicolumn{2}{|c|}{---------------Concentrações-----------. } \\
\hline & $0,5 \%$ & $1,0 \%$ \\
\hline Bioprotector $^{\circledR}$ & $0,0 \quad \mathrm{aA}^{*}$ & 0,0 aA * \\
\hline Canela & 0,0 aA * & $0,0 \mathrm{aA}^{*}$ \\
\hline Verbena & $0,0 \quad \mathrm{aA} *$ & $0,0 \mathrm{aA}^{*}$ \\
\hline Mostarda & $13,0 \mathrm{bB} *$ & $8,8 \mathrm{aA}^{*}$ \\
\hline Controle & \multicolumn{2}{|c|}{41,0} \\
\hline $\mathrm{CV}$ & \multicolumn{2}{|c|}{16,4} \\
\hline
\end{tabular}

Médias seguidas de mesma letra, minúscula na coluna e maiúscula na linha, não diferem entre si pelo teste de Skott-Knott em nível de $5 \%$ de probabilidade.

Médias seguidas de * diferem do tratamento controle em nível de $5 \%$ de probabilidade e médias seguidas de ${ }^{\text {NS }}$ não diferem do tratamento controle, ambos pelo teste de Dunnet.

Avaliação da qualidade fisiológica das sementes

Os tratamentos com Bioprotector $^{\circledR}$ $(0,5 \%)$ e óleo de mostarda $(0,5 \%)$ não afetaram o vigor das sementes (Tabela 3), entretanto, esses tratamentos apresentaram as maiores porcentagens de juvenis vivos (Tabela 1). Na concentração de $1,0 \%$, os tratamentos Bioprotector ${ }^{\circledR}$ e o óleo de canela mostraram-se mais tóxicos às sementes de $\boldsymbol{B}$. brizantha, proporcionando uma menor percentagem de sementes vigorosas, embora tenham sido os que mais reduziram o número de nematoides nessa dose.

Com exceção do produto Bioprotector ${ }^{\circledR}$ a $0,5 \%$ e do óleo de mostarda a $0,5 \%$ e $1,0 \%$, todos os tratamentos causaram redução do poder germinativo das sementes (Tabela 3). A redução observada pode ter ocorrido em função da maior dificuldade de embebição de água pelas sementes tratadas com óleos e/ou alteração dos processos fisiológicos envolvidos na germinação, por efeito químico.

Segundo ALMEIDA (1991), a germinação das sementes ou o desenvolvimento das plântulas de determinadas espécies sofre influência de aleloquímicos contidos em extratos e óleos vegetais. Todavia, no caso de plantas da Brassicaceae, família a qual pertence a mostarda, os mecanismos através dos quais os compostos alelopáticos influenciam a germinação, não são totalmente esclarecidos. O que se sabe é que os efeitos sobre a germinação ocorrem devido à decomposição dos glucosinolatos em isotiocianatos e tiocianatos (RIZZARDI, 2008). Esses compostos, em baixas concentrações, atrasam a germinação, mantendo as sementes viáveis e, em altas concentrações, podem penetrar em grandes quantidades, reagir irreversivelmente com enzimas e tornar as sementes inativas (PETERSEN et al., 2001). Como exemplo, pode-se citar o tratamento de sementes de picão-preto com extrato de folhas de canola (Brassica napus L.) que resultou em redução da germinação para 3\% (RIZZARDI, 2008). Entretanto, em determinadas situações, pode não haver alteração da qualidade fisiológica das sementes, como relatado por PAES (2012), que não constatou diminuição do poder germinativo de sementes de milho tratadas com óleo essencial de mostarda (contendo 90\% de isotiocianato alilo). Resultado semelhante foi observado neste trabalho, uma vez que o óleo de mostarda não inibiu a germinação das sementes de $\boldsymbol{B}$. brizantha.

O eugenol, composto presente no óleo de canela, provavelmente é um dos responsáveis pela inibição da germinação das sementes. Isto é coerente com os resultados obtidos por MAZZAFERA (2003), que relatou a inibição da germinação de sementes de algumas espécies vegetais pelos extratos de cravo-daíndia (rico em eugenol) e eugenol. Além do eugenol, o óleo de canela possui o ácido cinâmico, que, conforme ESPINDOLA et al. (2006), foi responsável pela inibição da germinação de sementes de tomate e redução da germinação de sementes de alface.

O óleo essencial de verbena possui alguns compostos como flavonoides, taninos e também eugenol, que inibem a germinação de algumas sementes. SANTOS \& REZENDE (2007) trataram sementes de plantas daninhas com flavonoides e observaram a inibição de sua germinação. Semelhantemente, SILVA (2007) observou redução da percentagem de germinação de sementes e do desenvolvimento de plântulas de sorgo e feijãoguandu após tratamento com extrato da casca de Mimosa tenuiflora (Wild) Poiret, espécie comum em regiões semi-áridas, que possui consideráveis teores de taninos. Dessa forma, esses mesmos compostos podem ter sido responsáveis pela redução da germinação das sementes de capim, quando tratadas com o óleo essencial de verbena, em ambas as concentrações.

A menor percentagem de nematoides recuperados está associada, em alguns tratamentos, à maior redução na germinação das sementes (Tabelas 1 e 3). Isso sugere que alguns compostos nematicidas podem também apresentar efeito alelopático. Todavia, o efeito observado pode ser resultado da maior embebição de outros compostos, contidos nos óleos, conjuntamente àqueles com ação nematicida. Essa redução foi mais acentuada nos tratamentos com 
Tabela 3 - Vigor e Germinação das sementes de Brachiaria brizantha cv. Marandu (\%) submetidas a tratamentos com óleos essenciais e o produto Bioprotector ${ }^{\circledR}$.

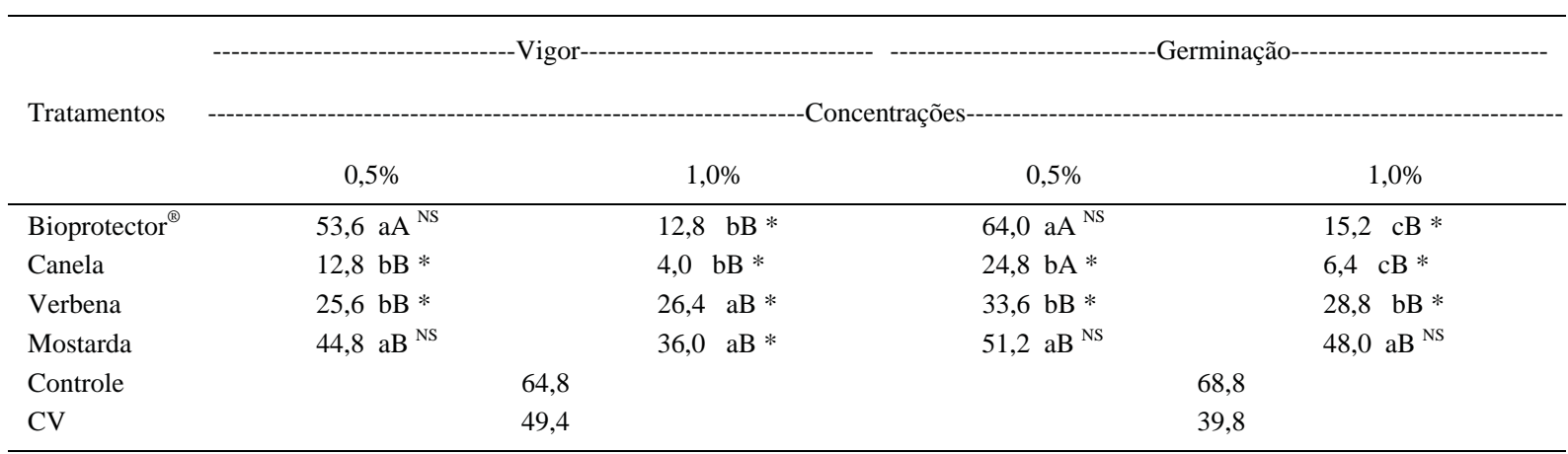

Médias seguidas de mesma letra, minúscula na coluna e maiúscula na linha, não diferem entre si pelo teste de Skott-Knott em nível de 5\% de probabilidade.

Médias seguidas de * diferem do tratamento controle em nível de $5 \%$ de probabilidade e médias seguidas de ${ }^{\mathrm{NS}}$ não diferem do tratamento controle, ambos pelo teste de Dunnet.

Bioprotector $^{\circledR}(1,0 \%)$, óleo de canela $(1,0 \%)$ e óleo de verbena $(1,0 \%)$.

Apesar das especulações sobre a ação de A. besseyi como causador de doenças em forrageiras, ainda não há quantificação dos danos causados por esse patógeno em Brachiaria spp. (BERNARD et al., 1998; PEDERSON \& QUESSENBERRY, 1998), sendo sua importância essencialmente quarentenária. Desse modo, o tratamento de sementes é fundamental para evitar a dispersão desse patógeno, que causa doenças em outras culturas. A aplicação de óleos essenciais é uma ferramenta potencial para utilização no manejo integrado desse nematoide.

\section{CONCLUSÃO}

Os óleos essenciais de verbena, canela e o produto Bioprotector ${ }^{\circledR}$ reduzem a população de $\boldsymbol{A}$. besseyi, em $B$. brizantha cv. 'Marandu', entretanto, não podem ser indicados para o tratamento dessas sementes por prejudicarem sua germinação.

O óleo de mostarda a $1,0 \%$ reduziu a população de $\boldsymbol{A}$. besseyi em sementes de $\boldsymbol{B}$. brizantha cv. 'Marandu' sem prejudicar significativamente sua germinação.

\section{AGRADECIMENTOS}

Ao Conselho Nacional de Desenvolvimento Científico e Tecnológico (CNPq), à Fundação de Amparo à Pesquisa do estado de Minas Gerais (Fapemig) e à Coordenação de Aperfeiçoamento de Pessoal de Nível Superior (CAPES), pelo apoio financeiro na execução do trabalho. A Cooperativa Agropecuária Pioneira LTDA, COOAPI, por ter cedido as sementes.

\section{REFERÊNCIAS}

ALMEIDA, F.S. Efeitos alelopáticos de resíduos vegetais. Pesquisa Agropecuária Brasileira, v.26, p.221-236, 1991. Disponível em: <http://webnotes.sct.embrapa.br/pdf/pab1991/ fevereiro/pab10_fev_91.pdf>. Acesso em: 05 jun. 2012.

ANDRÉS, M.F. et al. Nematicidal activity of essential oils: a review. Phytochemical Review, v.11, p.371-390, 2012 Disponível em: <http://link.springer.com/content/pdf/10.1007\%2 Fs11101-012-9263-3>. Acesso em: 03 mar. 2012. doi: 10.1007/ s11101-012-9263-3.

BAKKALI, F. et al. Biological effects of essencial oils-a review. Food Chemical Toxicology, v.46, p.446-475, 2008. Disponível em: <http:// link.springer.com/content/pdf/10.1007\%2Fs11101-012-9263-3>. Acesso em: 03 mar. 2012. doi: http: 10.1016/j.fct.2007.09.106.

BALA, S.K.; SUKUL, N.C. Systemic nematicide effect of eugenol. Nematropica, v.17, p.219-222, 1987.

BALDIN, E.L.L. et al. Uso de extratos vegetais, manipueira e nematicida no controle do nematoide das galhas em cenoura. Summa Phytopathologica, v.38, n.1, p.36-41, 2012. Disponível em: <http://www.scielo.br/scielo.php?pid=S010054052012000100006\&script=sci arttext $>$. Acesso em: 05 jun. 2012. doi: 10.1590/S0100-54052012000100006.

BERNARD, E.C. et al. Forage grasses. In: BARKER, K.R. et al. Plant and nematode interactions. Madison (EUA): American Society of Agronomy, 1998. p.427-454.

BRASIL. Ministério da Agricultura. Regras para análise de sementes. Brasília, Mapa/ACS, 2009. 200p.

CHRISTIE, J.R.; PERRY, V.G. Removing nematodes from soil. Proceedings of Helminthological Society of Washington, v.18, p.106-108, 1951.

ESPINDOLA, J.A.A. et al. Potencial alelopático de ésteres derivados do ácido cinâmico. Revista Floresta e Ambiente, v.13, p. 53-60, 2006.

Ciência Rural, v.44, n.7, jul, 2014. 
FERRAZ, S. et al. Extratos e óleos essenciais de plantas. In: Manejo sustentável de fitonematoides. Viçosa: UFV, 2010. p.170-186.

GARCIA, J.W.; TENENTE, R.C.V. Controle químico de Aphelenchoides besseyi Chistie em sementes de P. maximum. Nematologia Brasileira, v.25, p.95-98, 2001. Disponível em: <http://docentes.esalq.usp.br/sbn/nbonline/ol\%20251/95-98\%20 pb.pdf>. Acesso em: 05 jun. 2012.

KONG, J. et al. Nematicidal activity of Cassia and Cinnamon oil compounds and related compounds toward Bursaphelenchus xylophilus (Nematoda: Parasitaphelenchidae). Journal of Nematology, v.39, p.31-37, 2007. Disponível em: <http://www. ncbi.nlm.nih.gov/pmc/articles/PMC2586484/pdf/31.pdf>. Acesso em: 01 abr. 2013

MARCHI, C.E. et al. Nematofauna fitopatogênica de sementes comerciais de forrageiras tropicais. Pesquisa Agropecuária Brasileira, v.42, p.655-660, 2007. Disponível em: <http://www. scielo.br/pdf/pab/v42n5/07.pdf>. Acesso em: 05 jun. 2012. doi: 10.1590/S0100-204X2007000500007.

MAZAFERRA, P. Efeito alelopático do extrato alcoólico do cravo-da-índia e eugenol. Revista Brasileira de Botânica, v.26, p.231-238, 2003. Disponível em: <http://www.scielo.br/pdf/rbb/ v26n2/a11v26n2.pdf>. Acesso em: 05 jun. 2012. doi: 10.1590/ S0100-84042003000200011.

MONFORT, W.S. et al. Evaluating Brassica species as an alternative control measure for rootknot nematode (M. incognita) in Georgia vegetable plasticulture. Crop Protection, v.26, p.13591368, 2007. Disponível em: <http://www.sciencedirect.com/ science/article/pii/S026121940600367X>. Acesso em: 05 jun. 2012. doi: 10.1016/j.cropro.2006.11.008.

NEVES, W.S. et al. Ação nematicida de óleo, extratos vegetais e de dois produtos à base de Capsaicina, Capsainóides e Alil isotiocianato sobre juvenis de Meloidogyne javanica (Treub) Chitwood. Nematologia Brasileira, v.32, p.93-100, 2008. Disponível em: <http://docentes.esalq.usp.br/sbn/nbonline/ol\%20 322/93-100\%20co.pdf>. Acesso em: 05 jun. 2012.

PAES, J.L. et al. Insecticidal fumigant action of mustard essential oil against Sitophilus zeamais in maize grains. Crop Protection, v.34, p.56-58, 2012. Disponível em: <http://www.sciencedirect. com/science/article/pii/S0261219411003772>. Acesso em: 10 mar. 2013. doi: 10.1016/j.cropro.2011.11.021.

PETERSEN, J. et al. Weed supression by release of isothiocyanates from turnip-rap mulch. Agronomy Journal, v.93, p.37-43, 2001.

PEDERSON, G.A.; QUESENBERRY, K.H. Clovers and other forage legumes. In: BARKER, K.R. et al. Plant and nematode interactions. Madison: American Society of Agronomy, 1998. p.399-426.
RIZZARDI, M.A. et al. Potencial alelopático da cultura da canola (Brassica napus L. var. oleifera) na supressão de picão-preto (Bidens sp.) e soja. Revista Brasileira de Agrociência. v.14, n.2, p.239-248, 2008. Disponível em: <http://www.ufpel.edu.br/faem/ agrociencia/v14n2/artigo04.pdf>. Acesso em: 10 mar. 2013.

SANGWAN, N.K. et al. Nematicidal activity of essential oils of Cymbopogon grasses. Nematologica, v.31, p.93-99, 1985.

SANTOS, S.; REZENDE, M.O.O. Avaliação do potencial herbicida de compostos secundários na germinação de sementes de plantas daninhas encontradas em pastagens. Revista Analytica, v.32, p.72-78, 2007. Disponível em: <http://www.revistaanalytica. com.br/ed_anteriores/32/Art06.pdf>. Acesso em: 05 jun. 2012.

SILVA JUNIOR, G.J. et al. Efeito do produto Bioprotector sobre oídio (Sphaerotheca fuliginea) em pepino. In: CONGRESSO BRASILEIRO DE FITOPALOGIA, 40., 2007, Maringá, PR. Anais... Maringá: Tecart, 2007. p.298.

SILVA, W.A. Potencial alelopático de extratos do cumarú (Amburana cearensis A.C. Smith) e da jurema-preta (Mimosa tenuiflora (Willd.) Poir) na germinação e crescimento de sorgo (Sorghum bicolor L.), milho (Zea mays L.) e feijão guandu (Cajanus cajan L.). 2007. 62f. Dissertação (Mestrado em Zootecnia) - Curso de Sistemas Agrosilvipastoris no semi-árido, Universidade Federal de Campina Grande, Patos, PB.

SOUZA, F.H.D. Produção de sementes de gramíneas forrageiras tropicais. São Carlos: Embrapa Pecuária Sudeste, 2001. 40p. Acesso em: 05 jun. 2012. Online. Disponível em: <http://www.infoteca.cnptia.embrapa.br/bitstream/doc/45808/1/ PROCI2001.00030.pdf $>$.

TAIZ, L.; ZEIGER, E. Metabólicos secundários e defesa vegetal. In: _ Fisiologia vegetal. Porto Alegre: Artimed, 2004. p.309.

TENENTE, R.C.V.; MARQUES, A.S.A. Limpeza de sementes de $\boldsymbol{P}$. maximum infestadas com Aphelenchoides sp., através de tratamento físico e químico. In: REUNIÃO BRASILEIRA DE NEMATOLOGIA, 5., 1983, Brasília, DF. Anais... Brasília: Nematologia Brasileira, 1983. p.44.

TIMCHENKO, L.S.; MAIKO, T.K. Nematicidal properties of plants - antagonists of nematodes of decorative plants. Byulleten' Vsesoyuznogo Instituta Gel'mintologii im, v.50, p.81-84, 1989.

VECHIATO, M.H.; APARECIDO, C.C. Fungos em sementes de gramíneas forrageiras: restrição fitossanitária e métodos de detecção. Arquivos do Instituto Biológico, n.89, 2008. Acesso em: 05 jun. 2012. Online. Disponível em: <http://www.biologico. sp.gov.br/artigos_ok.php?id_artigo=89>. 